\title{
Epilog: In the Long Run We Are All Dead!
}

Sie haben dieses Buch nun von Anfang bis Ende durchgearbeitet und/oder haben immer wieder die Stellen aufgeschlagen, die für bestimmte Fragestellungen hilfreich sind. Ich hoffe sehr, dass ich Sie dabei unterstützen konnte, Ihre Gedanken zu ordnen, die Für und Wider bestimmter Fragestellungen abzuwägen.

Bei aller Planung in die Jahre hinein, bewahren Sie sich stets ein Mindestmaß an Flexibilität und Offenheit für Neues und Anderes. Denn das Leben hält immer die eine oder andere Überraschung bereit. Ich selbst habe mich mit Mitte Dreißig dazu entschlossen, einen ganz anderen Weg einzuschlagen als die Jahre zuvor. Und auch zu Beginn meines Studiums der Soziologie hätte ich nicht gedacht, dass ich nach Abschluss des Studiums die ersten Berufsjahre als Planer und Controller in einer Bank verbringen würde, um dann über das Human Resources Management und die soziale Arbeit zurück an die Universität zu finden.

Planung ist wichtig. Aber je weiter Sie in die Zukunft planen, desto mehr sollten Sie mein Lieblingszitat des Wirtschaftsnobelpreisträgers John Maynard Keynes im Hinterkopf behalten: „In the long run we are all dead.“

Wie eingangs erwähnt, ist das Buch aus meiner beratenden Arbeit mit Studierenden heraus entstanden. Ganz in diesem Sinne freue ich mich, wenn weitere Impulse aus Ihren Reihen in eine zweite Auflage einfließen: Was fanden Sie gut? Was haben Sie sich anders vorgestellt? Welche Fragen/Informationen haben Ihnen gefehlt?

Schreiben Sie mir: stephan.pflaum@lmu.de 